\title{
Nutrição em vão
}

\section{Dr. Fabiano de Abreu Agrela Rodrigues}

deabreu.fabiano@gmail.com

$\mathrm{PhD}$, neurocientista, psicanalista, biólogo, historiador e antropólogo

\section{RESUMO}

Os alimentos possuem diferentes nutrientes e em cada um deles ou em conjunto, promovem benefícios únicos. A principal fonte de nutrientes para o organismo ter um funcionamento adequado tanto físico quanto mental é por meio da alimentação. Uma pessoa com alimentação saudável evita algumas doenças como: obesidade, câncer, artrite, anemia, diabetes, hipertensão e muitas outras ou a maioria delas. O objetivo do presente estudo é compreender se estamos consumindo uma quantidade maior de alimentos sem necessidade e se isso será prejudicial no futuro já que, o organismo mediante ao processo evolutivo "aprendeu" a armazenar e só utilizar o necessário. O presente estudo trata-se de uma revisão de literatura no portal de periódicos eletrônicos da SciELO, PubMed e Science Direct. Concluindo assim, que apesar de haver uma grande quantidade de alimentação para toda a população, quando há um consumo exagerado, apesar desses alimentos possuírem nutrientes importantes, podem trazer malefícios a nossa saúde.

Palavras-chave: nutrição; alimentação; estilo de vida; nutrientes. 


\title{
Nutrition in vain
}

\begin{abstract}
Foods have different nutrients and in each of them or together, they provide unique benefits. The main source of nutrients for the body to function properly, both physically and mentally, is through food. A person with healthy diet avoids some diseases such as: obesity, cancer, arthritis, anemia, diabetes, hypertension and many others or most of them. The aim of this study is to understand if we are consuming a greater amount of food unnecessarily and if this will be harmful in the future since the organism, through the evolutionary process, "learned" to store and only use what is necessary. of a literature review in the portal of electronic journals of SciELO, PubMed and Science Direct. Thus, concluding that, despite a large amount of food for the entire population, when there is an exaggerated consumption, despite these foods having important nutrients, they can bring harms our health.
\end{abstract}

Keywords: nutrition, food, lifestyle, nutrients. 


\section{INTRODUÇÃO}

\subsection{Importância da alimentação}

Os benefícios de uma alimentação saudável é a prevenção e tratamento de doenças, melhora do humor, concentração, etc. Diminuição e manutenção do peso; devido a reeducação alimentação para equilíbrio da saúde (Ścieszka, 2019).

Aumento da disposição; quando o indivíduo se alimenta adequadamente, é obtido uma melhora na disposição do organismo para que realize tarefas do dia a dia. Alimentos ricos em carboidratos, são a principal fonte imediata de energia e força. Melhora do humor; devido a ingestão de alimentos que estimulam a serotonina como o chocolate. $\mathrm{O}$ espinafre, possuí vitamina B e ácido fólico, tais substâncias atuam no sistema nervoso e contribuem para a diminuição do estresse (Schwingshackl, 2017).

Fortalecem os ossos; alimentos ricos em cálcio e ferro quando associados possuem uma grande influência no fortalecimento dos ossos, como exemplo de alimentos como salmão, sardinha, espinafre, oleaginosas, vegetais verde escuro, leite e seus derivados. Alguns alimentos, regulam o organismo, no déficit nutricional, ganho ou perda de peso em excesso, doenças, metabolismo lento, entre outros (Schwingshackl, 2017).

Os alimentos possuem diferentes nutrientes e é cada um deles ou em conjunto que promoverá seus benefícios únicos; sendo divididos em macronutrientes e micronutrientes (Schwingshackl, 2017).

Os macronutrientes são os carboidratos que possuem função principal de proporcionar energia ao organismo, como um combustível para que o indivíduo possa realizar atividades do dia a dia. Os principais alimentos fontes de carboidratos são: batata-doce, ervilhas e grão de bico cozidas, arroz, pão, cereais, dentre outros (San-Cristobal, 2020). Os lipídios são moléculas orgânicas insolúveis em água e solúveis em certas substâncias orgânicas, também são responsáveis pelo fornecimento de energia, é a maneira que o corpo encontra para armazenar o excesso de energia obtido através da alimentação. Os principais alimentos fontes de lipídios são: abacate, linhaça, carne bovina, coco, manteiga, chocolate amargo, azeite de oliva, dentre outros (San-Cristobal, 2020).

As proteínas atuam na estruturação das células, atuando em quase todos os processos vitais. Suas funções são específicas para cada uma delas, auxiliam para que a célula tenha sua integridade mantida, defendê-la de agentes externos, reparar danos, controlar e regular funções celulares. Os principais alimentos fontes de proteínas são: soja, 
camarão, frango, salmão, amêndoa, carne vermelha e peixes, dentre outros (SanCristobal, 2020).

Já os micronutrientes são vitaminas, substâncias orgânicas fundamentais para o corpo humano, sendo necessário em pequenas quantidades. Atuam de maneira essencial para o sistema imunológico e funcionamento adequado do metabolismo. Os principais alimentos fontes de vitaminas são; morango, kiwi, laranja, brócolis, couve-flor, dentre outros (Kozeniecki, 2020).

Os sais minerais desempenham diversas funções no organismo tais como, produção de hormônios a formação de dentes, ossos, regulação da pressão sanguínea, dentre outras funções especificas de cada um deles. Os principais alimentos fontes de sais minerais são: carnes, porco, frango, repolho, banana, laranja, entre outros (Kozeniecki, 2020).

As fibras que alguns autores não as classificam como nutrientes, atuam no desempenho do intestino, diminuem a absorção de colesterol, gordura e açúcar. Os principais alimentos fontes de fibras são: feijões, pães, biscoitos integrais, milho, entre outros (Chen, 2017).

Nosso organismo é capaz de armazenar os carboidratos, lipídios, sais minerais e vitaminas. Entretanto, não é capaz de armazenar fibras e proteínas (Chen, 2017).

\subsection{Estilo de vida geral}

A alimentação brasileira possuí origens portuguesa, indígena e africana. Devido as imigrações, italiana, japonesa, alemã, árabe e outros no final do século XIX houveram outras influencias no cardápio do brasileiro, porém deste modo também teriam de se adaptar aos ingredientes disponíveis no pais (Santana, 2020).

Em cada região houveram rastros dessas origens. Região norte influenciada pelos indígenas possuí uma riqueza em sua gastronomia de alimentos vindos de recursos naturais, como mandioca-brava para formar farinhas, peixe pirarucu, guaraná, castanhado-pará, pimentas e outros. Alimentos esses ricos em antioxidantes, auxiliam na regulação da pressão arterial, obesidade, e taxas de colesterol (Santana, 2020).

Na região nordeste; há muitos frutos do mar, variedades de frutas, carne-seca (influência africana) tapioca, azeite-de-dendê, queijo coalho, castanha-de-caju e o acarajé que é rico em vitamina A e E, devido a seus efeitos antioxidantes, auxíliam no envelhecimento precoce, porém não é indicado seu consumo em excesso, devido ao seu teor de sódio (Ramalho, 2020). 
Hábitos alimentares na região Centro-Oeste: estão concentrados três biomas, Amazônia, Cerrado e Pantanal, ricos em uma variedade de vegetação e recursos hídricos. Os ingredientes encontrados são: pequi, peixes de água doce, mandioca, ervas, banana-daterra e o frango. rico em vitaminas B3, B6, selênio dentre outros, atuando na dilação dos vasos sanguíneos, sua carência, leva a fraqueza muscular e lesões na pele. Porém seu excesso, pode baixar os níveis de colesterois, prejudicando o organismo (Ramalho, 2020).

Região Sudeste, recebeu diversos imigrantes que influenciaram nas tradições culinárias da região. Ingredientes: porco, leilão, cuscuz, queijo de minas, milho-verde, torresmo, massas, pizzas e o milho que atua na saúde intestinal, visão e quando consumido em excesso, é possível que haja acúmulo de açucar no sangue (Ramalho, 2020). Na região sul, onde possuí um clima mais frio, devido a tradição europeia. Ingredientes: erva-mate, chimarrão, churrasco, uvas, vinhos.

\section{OBJETIVO}

- Compreender se estamos consumindo uma quantidade maior de alimentos sem necessidade e se isso será prejudicial no futuro.

\section{METODOLOGIA}

O presente estudo trata-se de uma revisão de literatura no portal de periódicos eletrônicos da SciELO, PubMed e Science Direct. Sendo utilizado os seguintes termos para realização da busca de dados em português: nutrição, alimentação, estilo de vida, nutrientes, fome e em inglês: nutrition, food, lifestyle, nutrients, hunger.

\section{RESULTADOS}

\subsection{Estilo de vida de pessoas que vivem mais Nutrição, tempo de vida das que mais passam fome.}

O brasileiro vive em média até os 76,3 anos. Em 1940, a média era de 45,5 anos (Almeida, 2019). No ano de 2016, de acordo com a OMS (Organização Mundial da Saúde, as DCNTs (Doenças crônicas não transmissíveis), 74\% das mortes totais no pais foram decorrentes de: diabetes, doenças cardiovasculares e obesidade, por exemplo, que possuem grande influência no consumo de nutrientes (Lee, 2018).

Sendo assim, algumas mudanças nos hábitos alimentares são capazes de aumentar consideravelmente a expectativa de vida destes indivíduos (Lee, 2018).

Em relação ao tipo de alimentos relatados pelas pessoas que vivem por mais tempo, a 
maioria prioriza os naturais como; legumes, verduras, frutas, grãos. Possuem um consumo de carne vermelha menor, deixando para ocasiões especiais. Em contrapartida o consumo de carne branca (frango e peixes) são maiores. Há uma moderação nos alimentos lácteos derivados ao leite de vaca, porém de ovelha e cabra não há restrições (Lee, 2018).

Em um estudo realizado por Tsugane et. al. 2021, tentou entender o porquê de a população japonesa viver mais do que as demais. Verificando que o baixo consumo de carne vermelha, ácidos graxos saturados e alta ingestão de peixes, sem haver exagero no consumo de alimentos, melhora a qualidade de vida, dessa população.

Um estudo desenvolvido entre os anos de 1992 e 2013 em 164 países, foi avaliado o consumo excessivo de carne vermelha e a sua influência na qualidade de vida dos indivíduos. Demonstrando que países com alto índice de consumo de carne vermelha desenvolvem um impacto negativo na longevidade de vida desses indivíduos (Ranabhat, 2020).

\subsection{Influência da alimentação no cérebro}

A carne vermelha, possuí nutrientes importantes para o organismo como a vitamina B3 que atua nas reações de produção de energia pelas células, em uma porção, é possível obter 5,0 mg e seu armazenamento ocorre no estômago e intestino delgado. Porém seu excesso pode levar a doenças como; diabetes, pressão baixa, gota, alergias, úlceras, problemas na vesícula, no fígado, no coração e nos rins (Egelandsdal, 2020).

A B6 e B12 presentes na formação dos glóbulos vermelhos e funcionamento adequado do sistema nervoso. Sendo a primeira, absorvida em $0,52 \mathrm{mg}$ no intestino delgado em excesso pode danificar os nervos (neuropatia) gerando dor e dormência nos pés e nas pernas e a B12, que quando consumida, 100 gramas de carne, são absorvidos cerca de 2,5 mg de vitamina B12, sendo a carne a principal fonte desta vitamina e em sua maioria é absorvida e armazenada, $60 \%$ no fígado e $30 \%$ nos músculos, até que seja necessária ao organismo, falta pode gerar anemia e seu excesso pode levar a reações alérgicas ou aumento do risco de infecções (Wang, 2019).

Devido ao fato da carne vermelha ser rica em nutrientes, traz diversos benefícios a saúde mental, como por exemplo na produção de neurotransmissores como serotonina, dopamina, noradrenalina, acetilcolina, ácido gama-aminobutírico (GABA), atuantes na comunicação entre os neurônios, sendo responsáveis pelo prazer, medo, bom humor, 
memória, aprendizado, alerta, entre outros. A falta de nutrientes pode prejudicar tais neurotransmissores, favorecendo o surgimento de doenças como, depressão, distúrbio de ansiedade, Parkinson, mau-humor, dificuldades de aprendizagem, memória ruim (Jawhara, 2020).

Em um ensaio clínico desenvolvido por Formica et. al. 2020, com uma amostra de 154 participantes avaliou os efeitos do consumo de carne vermelha, 3 dias na semana. Apresentando assim, resultados satisfatórios na função cognitiva de tais indivíduos.

Porém, o seu excesso pode acarretar no aumento do risco de doenças como infarto, insuficiência cardíaca, câncer, níveis de colesterol dentre outras (Koeth, 2019).

As nozes da mesma família da avelã e castanhas, rico em nutrientes como vitamina B6, ômega 3 e 6 . A vitamina $\mathrm{C}$ que é absorvida principalmente no osso ilío e no intestino delgado, através do sangue é levada a todos os tecidos, o que não é utilizada é eliminado na urina. Vitamina E que é armazenada no fígado, tecido adiposo, coração, músculos, testículos, útero, sangue, glândulas suprarrenais e glândula pituitária em excesso pode levar a hemorragia, bem como fraqueza muscular, fadiga, náuseas e diarreia(Amarowicz, 2020).

Devido a suas concentrações de vitamina E e ômega-3, atuam no processo de envelhecimento dos neurônios e atuando no retardo da perca de memória (Amarowicz, 2020).

Em um estudo com 636 idosos, que obtiveram uma dieta rica em nozes durante o período de dois anos, revelando efeitos benéficos nas funções cognitivas destes indivíduos (SalaVila, 2020). Corroborando com outro estudo, que avaliou 166 idosos durante o período de 6 meses, o primeiro grupo recebeu dieta rica com nozes e o segundo alimentação normal. Apresentando havendo melhoras no desempenho cognitivo do grupo tratado, como melhora velocidade de processamento, memória, atenção, funções executivas, capacidade visual espacial e visuomotora (Knight, 2015).

Apesar de trazer diversos benefícios ao organismo, quando consumida em excesso, devido a ser rica em gorduras, contribui ao aumento do peso corporal, níveis de colesterol, favorecendo doenças como: infarto, insuficiência cardíaca dentre outros (Kim, 2016).

O triptofano, aminoácido essencial, que quando associado a vitamina B3, atua favorecendo o cérebro, na produção de serotonina, neurotransmissor importante na 
regulação do humor, bem-estar, felicidade. Após sua absorção atravessa a barreira hematoencefálica. Além disso, ajuda no alívio da depressão e ansiedade, dor, déficit de atenção, hiperatividade, fadiga crônica e tensão pré-menstrual (TPM). Tal substância é encontrada em diversos alimentos como: peixes, pão e arroz integral, queijo, ovo, castanhas, banana e leite (Strasser, 2016).

Em excesso pode gerar azia, dor de estômago, enjoos, vômitos, gases, diarreia, perda de apetite, tonturas, dores de cabeça, boca seca, fraqueza muscular e sonolência (Khanipour, 2019).

Em um ensaio clínico randomizado controlado, com 40 indivíduos do sexo masculino, ambos com déficit de atenção. O primeiro grupo obteve uma bebida com triptofano, enquanto o segundo não. Evidenciando assim, uma pequena melhora dos níveis de atenção pelo grupo tratado (Zimmermann, 2012).

Porém seu excesso pode levar a efeitos colaterais como azia, dor de estômago, enjoos, vômitos, gases, diarreia, perda de apetite, tonturas, dores de cabeça, boca seca, fraqueza muscular e sonolência excessiva (Strasser, 2016).

\subsection{Relação dos nutrientes com o ácido desoxirribonucleico (DNA) e memória ancestral}

Devido ao fato do nosso corpo poder armazenar a grande maioria dos nutrientes essenciais para serem utilizados posteriormente, quando necessário estamos sempre em consumo e promovendo um acúmulo muito maior do que o essencial (Gombart, 2020). Caso isso ocorra com frequência, o DNA irá memorizar este acúmulo de nutrientes em excesso a não necessidade, fazendo assim com que sejamos dependente de nutrientes que antes armazenávamos devido a um período ancestral mais escasso de alimentos, sendo assim nosso organismo possuí uma memória ancestral referente a tais armazenamentos, podendo ser prejudicial no futuro (González, 2020).

A patologia neuropsiquiátrica fenilcetonúria clássica, é provocada devido a mutações no gene da enzima fenilalanina hidroxilase, responsável por processar o aminoácido fenilalanina. E seu tratamento é justamente a diminuição da ingestão do aminoácido fenilalanina (Lichter-Konecki, 2019).

\section{CONSIDERAÇÕES FINAIS}

Apesar da alta demanda de nutrientes suprindo assim grande parte da população com condição financeira, quando há o consumo exagerado, mesmo que os alimentos possuam 
importantes nutrientes, há possibilidade de trazer malefícios para a saúde.

Os alimentos em grande quantidade exigem um esforço maior do corpo, esgotando-o, comprometendo a longevidade. Nosso organismo absorve o necessário e o excesso é eliminado e causam danos a saúde.

Também analiso a relação com a adaptação ao longo da evolução. Nosso organismo está adaptado ao armazenamento para uso posterior; se suprirmos sempre, o DNA vai memorizar a não necessidade, fazendo com que sejamos dependente de nutrientes que antes armazenávamos devido a um período passado mais escasso de alimentos.

O uso excessivo de suplementação pode afetar a saúde pelo excesso no presente e causar danos à saúde no futuro.

\section{REFERÊNCIAS}

ALMEIDA, M.A., Almeida-Silva, F., Guimarães, A.J., Almeida-Paes, R., ZancopéOliveira, R.M. The occurrence of histoplasmosis in Brazil: A systematic review. Int J Infect Dis. n. 86, págs. 147-156, 2019 doi: 10.1016/j.ijid.2019.07.009.

AMAROWICZ, R., Pegg, R.B. Tree Nuts and Peanuts as a Source of Natural Antioxidants in our Daily Diet. Curr Pharm Des. n. 26, v. 16, págs. 1898-1916, 2020 doi: 10.2174/1381612826666200318125620.

CHEN JP, Chen GC, Wang XP, Qin L, Bai Y. Dietary Fiber and Metabolic Syndrome: A Meta-Analysis and Review of Related Mechanisms. Nutrients. 2017 Dec 26;10(1):24. doi: 10.3390/nu10010024.

EGELANDSDAL, B., Oostindjer, M., Hovland, E.M., Okholm, B., Saarem, K., Bjerke, F., Ruud, L., Grabež, V., Haug, A. Identifying labelling and marketing advantages of nutrients in minced beef meat: A case study. Meat Sci. n. 159, e107920, 2020 doi: 10.1016/j.meatsci.2019.107920

FORMICA, M.B., Gianoudis, J., Nowson, C.A., O'Connell, S.L., Milte, C., Ellis, K.A., Daly, R.M. Effect of lean red meat combined with a multicomponent exercise program on muscle and cognitive function in older adults: a 6-month randomized controlled trial. Am J Clin Nutr. n.134, v. 1, págs. 113-128, 2020 doi: 10.1093/ajen/nqaa104.

Gombart AF, Pierre A, Maggini S. A Review of Micronutrients and the Immune SystemWorking in Harmony to Reduce the Risk of Infection. Nutrients. 2020 Jan 16;12(1):236. doi: 10.3390/nu12010236. 
González A, Hall MN, Lin SC, Hardie DG. AMPK and TOR: The Yin and Yang of Cellular Nutrient Sensing and Growth Control. Cell Metab. 2020 Mar 3;31(3):472-492. doi: 10.1016/j.cmet.2020.01.015.

JAWHARA, M., Sørensen, S.B., Heitmann, B.L., Halldórsson, P.I., Pedersen, A.K., Andersen, V. The Relation between Red Meat and Whole-Grain Intake and the Colonic Mucosal Barrier: A Cross-Sectional Study. Nutrients. n. 12, v. 6, e1765, 2020 doi: 10.3390/nu12061765.

KHANIPOUR, S., Mehri, M., Bagherzadeh-Kasmani, F., Maghsoudi, A., Assadi, S.E. Excess dietary tryptophan mitigates aflatoxicosis in growing quails. J Anim Physiol Anim Nutr (Berl). n. 103, v. 5, págs. 1462-1473, 2019 doi: 10.1111/jpn.13167.

KIM, Y., Keogh, J.B., Clifton, P.M. Differential Effects of Red Meat/Refined Grain Diet and Dairy/Chicken/Nuts/Whole Grain Diet on Glucose, Insulin and Triglyceride in a Randomized Crossover Study. Nutrients. n. 30, v. 11, págs. :687, 2016 doi: $10.3390 /$ nu8110687.

KNIGHT, A., Bryan, J., Wilson, C., Hodgson, J., Murphy, K. A randomised controlled intervention trial evaluating the efficacy of a Mediterranean dietary pattern on cognitive function and psychological wellbeing in healthy older adults: the MedLey study. BMC Geriatr. Apr n. 28, v. 15, 2015 doi: 10.1186/s12877-0150054-8

KOETH, R.A., Lam-Galvez, B.R., Kirsop, J., Wang, Z., Levison, B.S., Gu, X., lCarnitine in omnivorous diets induces an atherogenic gut microbial pathway in humans. J Clin Invest. n. 129, v. 1, págs. 373-387, 2019 doi: 10.1172/JCI94601.

KOZENIECKI, M., Ludke, R., Kerner, J., Patterson, B. Micronutrients in Liver Disease: Roles, Risk Factors for Deficiency, and Recommendations for Supplementation. Nutr Clin Pract. n. 35, v. 1, págs. 50-62, 2020 doi: 10.1002/ncp.10451.

LEE, E.S., Song, E.J., Nam, Y.D., Lee, S.Y. Probiotics in human health and disease: from nutribiotics to pharmabiotics. J Microbiol. n. 56, v. 11, págs. 773-782, 2018 doi: 10.1007/s12275-018-8293-y.

LICHTER-KONECKI U, Vockley J. Phenylketonuria: Current Treatments and Future Developments. Drugs. 2019 Apr;79(5):495-500. doi: 10.1007/s40265-019- 
01079-z

RAMALHO, A.A., Holanda, C.M., Martins, F.A., Rodrigues, B.T.C., Aguiar, D.M., Andrade, A.M., Koifman, R.J. Food Insecurity during Pregnancy in a MaternalInfant Cohort in Brazilian Western Amazon. Nutrients. n. 28, 6, e12 pág. 1578, 2020 doi: 10.3390/nu12061578.

RANABHAT, C.L., Park, M.B., Kim, C.B. Influence of Alcohol and Red Meat Consumption on Life Expectancy: Results of 164 Countries from 1992 to 2013. Nutrients. n.12, v. 2, e12 pág. 459, 2020 doi: 10.3390/nu12020459.

SALA-VILA A., Valls-Pedret, C., Rajaram, S., Coll-Padrós, N., Cofán, M., Serra-Mir, M., Pérez-Heras, A.M., Roth, I., Freitas-Simoes, T.M., Doménech, M., Calvo, C., Effect of a 2-year diet intervention with walnuts on cognitive decline. The Walnuts And Healthy Aging (WAHA) study: a randomized controlled trial. Am J Clin Nutr. n.1111, v. 3, págs. 590-600, 2020 doi: 10.1093/ajcn/nqz328.

SAN-CRISTOBAL, R., Navas-Carretero, S., Martínez-González, M.Á., Ordovas, J.M., Martínez, J.A. Contribution of macronutrients to obesity: implications for precision nutrition. Nat Rev Endocrinol. n. 16, v. 6 págs. 305-320, 2020 doi: 10.1038/s41574-020-0346-8.

SANTANA, A.B.C, Sarti, F.M. Avaliação dos indicadores de aquisição, disponibilidade e adequação nutricional da cesta básica de alimentos brasileira [Assessment of the indicators of purchasing, availability, and nutritional adequacy of the Brazilian basic food basket]. Cien Saude Colet. n. 25, v. 10, págs. 4001-4012, 2020 Portuguese. doi: 10.1590/1413-812320202510.35192018.

SCHWINGSHACKL, L., Hoffmann, G., Lampousi, A.M., Knüppel, S., Iqbal, K., Schwedhelm, C., Bechthold, A., Schlesinger, S., Boeing, H. Food groups and risk of type 2 diabetes mellitus: a systematic review and meta-analysis of prospective studies. Eur J Epidemiol. n. 32, v. 5 págs. 363-375, 2017 doi: 10.1007/s 10654-017-0246-y.

ŚCIESZKA, S., Klewicka, E. Algae in food: a general review. Crit Rev Food Sci Nutr. n. 59, v. 21 págs. 3538-3547, 2019 doi: 10.1080/10408398.2018.1496319.

STRASSER, B., Geiger, D., Schauer, M., Gostner, J.M., Gatterer, H., Burtscher, M., Fuchs, D. Probiotic Supplements Beneficially Affect Tryptophan-Kynurenine Metabolism and Reduce the Incidence of Upper Respiratory Tract Infections in 
Trained Athletes: A Randomized, Double-Blinded, Placebo-Controlled Trial. Nutrients. n. 23, v. 11, 2016 doi: 10.3390/nu8110752.

TSUGANE, S. Why has Japan become the world's most long-lived country: insights from a food and nutrition perspective Eur J Clin Nutr. n. 75, v. 6, págs. 921-928, 2021 doi: 10.1038/s41430-020-0677-5.

WANG, Z., Bergeron, N., Levison, B.S, Li, X.S., Impact of chronic dietary red meat, white meat, or non-meat protein on trimethylamine N-oxide metabolism and renal excretion in healthy men and women. Eur Heart J. n. 40, v. 7, págs. 583594, 2019 doi: 10.1093/eurheartj/ehy799.

ZIMMERMANN, M., Grabemann, M., Mette, C., Abdel-Hamid, M., Uekermann, J., Kraemer, M., Wiltfang, J., Kis, B., Zepf, F.D. The effects of acute tryptophan depletion on reactive aggression in adults with attention-deficit/hyperactivity disorder (ADHD) and healthy controls. PLoS One. 2012 n. 7, v. 3, e32023, 2012 doi: 10.1371/journal.pone.0032023. 